\title{
Effective stretching position for the supraspinatus muscle evaluated by shear wave elastography in vivo
}

\section{AUTHOR(S):}

Nishishita, Satoru; Hasegawa, Satoshi; Nakamura, Masatoshi; Umegaki, Hiroki; Kobayashi, Takuya; Ichihashi, Noriaki

\section{CITATION:}

Nishishita, Satoru ... [et al]. Effective stretching position for the supraspinatus muscle evaluated by shear wave elastography in vivo. Journal of shoulder and elbow surgery 2018, 27(12): 2242-2248

\section{ISSUE DATE:}

2018-12

URL:

http://hdl.handle.net/2433/245812

\section{RIGHT:}

(c) 2018. This manuscript version is made available under the CC-BY-NC-ND 4.0 license

http://creativecommons.org/licenses/by-nc-nd/4.0/; The full-text file will be made open to the public on 1 December 2019 in accordance with publisher's 'Terms and Conditions for Self-Archiving'.; この論文は出版社版でありません。引 用の際には出版社版をご確認ご利用ください。; This is not the published version. Please cite only the published version. 
2 Effective stretching position for the supraspinatus muscle evaluated by shear wave

3 elastography in vivo

$5 \quad$ Authors and academic degrees

6 Satoru Nishishita, P.T., M.Sc. 1) 2) 3), Satoshi Hasegawa, P.T., Ph.D. ${ }^{1)}$, Masatoshi Nakamura,

7 P.T., Ph.D. ${ }^{1)}$, Hiroki Umegaki, P.T., M.Sc. ${ }^{1)}$, Takuya Kobayashi, P.T., M.Sc. ${ }^{1)}$, Noriaki

8 Ichihashi, P.T., Ph.D. ${ }^{1)}$

9

\section{Department and Institution}

$11{ }^{1)}$ Human Health Sciences, Graduate School of Medicine, Kyoto University

$12^{2)}$ Institute of Rehabilitation Science, Tokuyukai Medical Corporation

3) Kansai Rehabilitation Hospital, Tokuyukai Medical Corporation

*Corresponding author:

16 Satoru Nishishita

17 Human Health Sciences, Graduate School of Medicine, Kyoto University

1853 Shogoinkawahara-cho, Sakyo-ku, Kyoto 606-8397, Japan

19 Telephone: +81-75-751-3935; Fax: +81-75-751-3909 
20 E-mail: nishishitasatoru@gmail.com

21

\section{Ethical Committee approval}

23 This study has been approved by the Ethics Committee of the Kyoto University

24 Graduate School and Faculty of Medicine: No. R0314.

25

26 Acknowledgements

27 We would like to thank Ms. Ibuki and Editage (www.editage.jp) for English language editing.

28 This study was supported by the Grant - in - Aid for Scientific Research (B) 15 H03043.

29

30 Conflict of Interest

$31 \quad$ None.

32 


\section{Abstract}

\section{Background:}

35 Stretching is useful for increasing flexibility in clinical and athletic situations. Although

36 several authors have recommended various stretching techniques for the supraspinatus

37 muscle, there is no consensus on the effective stretching position owing to a lack of quantitative analysis in vivo. This study used ultrasonic shear wave elastography in vivo to verify the effective stretching positions for the supraspinatus muscle.

\section{Methods:}

The study participants were 15 healthy male volunteers. The shear elastic modulus, used as the index of supraspinatus muscle elongation, was computed using ultrasonic shear wave elastography. The shear elastic modulus was measured at neutral position and maximum internal rotation in 9 positions: $0^{\circ}$ elevation, $90^{\circ}$ abduction, $90^{\circ}$ flexion, maximum extension, maximum horizontal adduction at $45^{\circ}$ and $90^{\circ}$ elevation, and maximum horizontal abduction at $20^{\circ}, 45^{\circ}$, and $90^{\circ}$ elevation.

47 Results:

48 The shear elastic moduli were significantly greater in maximum internal rotation at maximum horizontal abduction with $45^{\circ}$ and $90^{\circ}$ elevation and maximum internal rotation at maximum extension than those in the other positions. There were no significant differences in the shear elastic moduli among these 3 positions.

\section{Conclusions:}

53 This study demonstrated that maximum internal rotation at maximum extension, maximum internal rotation at maximum horizontal abduction with $90^{\circ}$ elevation, and maximum internal rotation at maximum horizontal abduction with $45^{\circ}$ elevation are effective stretching positions for the supraspinatus muscle. 


\section{$58 \quad$ Keywords}

$59 \quad$ ultrasonic shear wave elastography

60 shear elastic modulus

61 supraspinatus muscle

62 stretching

63 shoulder

64 rehabilitation

65

\section{Level of evidence}

67 Basic Science Study, Biomechanics, Imaging.

68 


\section{Introduction}

Stretching is useful for increasing flexibility in clinical and athletic situations. Many previous studies have reported on the effects of stretching ${ }^{7,9,11,18,26}$ but few studies have reported the method or position used to create an effective stretch ${ }^{24,30}$. Because the shoulder joint has multiple degrees of freedom and a large range of motion, the method used to stretch shoulder muscles needs to be investigated.

Many studies have reported the relationship between the 3-dimensional shoulder position and the moment $\operatorname{arm}^{16,17,32}$ and torque-vector directions ${ }^{2,37}$ of each shoulder muscle.

Therefore, the 3-dimensional shoulder position must be considered when devising effective methods for stretching the shoulder muscles.

Several authors have recommended various stretching positions for each individual muscle $e^{6,10,31,36}$, but there is no consensus on the effective stretching positions owing to a lack of an in vivo quantitative analysis. The cross-body stretch and the sleeper stretch are well known and commonly used for posterior shoulder tightness ${ }^{22}$, but the effect of stretching on individual muscles and other tissues is unclear.

In previous cadaveric studies, the effective stretching position for the shoulder muscles and joint capsule was simulated and quantitatively analyzed ${ }^{13,23-25}$. Clinicians are in great need of an in vivo quantitative analysis of the effect of stretching on individual muscles, but conventionally, it has been difficult to measure the evaluation index of stretching on individual muscles. In human studies, passive torque-angle measurements are widely used to noninvasively examine muscle stretch and passive muscle force $\mathrm{e}^{27,33,34}$. However, torque-angle measurements are affected by many structures crossing the joint, such as synergistic muscles, aponeuroses, tendons, joint capsules, and ligaments, and cannot be used to identify the effect of an individual muscle. Therefore, passive torque-angle measurements 
93 are not specific to the passive stretching response of individual muscles, especially for the

94

95

96

97

98

99

100

101

102

103

104

105

106

107

108

109

110

111

112

113

114

115 muscles of the shoulder joint.

A new ultrasound-based technology, called ultrasonic shear wave elastography, has been developed that reliably and noninvasively measures soft tissue viscoelastic properties ${ }^{1}$. Many studies have quantitatively assessed the muscle shear elastic modulus in vivo and in vitro ${ }^{4,14}$, $15,19,21$

The occurrence of shoulder injuries are associated with the supraspinatus (SSP) muscle and infraspinatus muscle because these muscles contribute to the dynamic stability of the shoulder joint ${ }^{35}$. We targeted the SSP because there is more evidence of reliability and validity using elastography on measuring the $\mathrm{SSP}^{8,12,28}$ rather than infraspinatus muscle. Specifically, researchers have reported the link between a tight SSP and abduction contracture ${ }^{5}$.

Several authors have recommended effective stretching positions for the SSP based on their knowledge of anatomy and kinesiology 6 , 10, 31, 36. The positions recommended for stretching the SSP are fully adducting the arm behind the back ${ }^{6}$, positioning the arm behind the back while maintaining medial rotation ${ }^{10}$, extension, adduction, and internal rotation $(\mathrm{IR})^{36}$, and placing the hand behind the back and reaching up between the shoulder blades ${ }^{31}$.

Despite these recommendations, there is no consensus on the effective stretching positions.

One cadaveric study recommended positioning the arm at abduction with extension as the most effective stretching position for the $\mathrm{SSP}^{24}$. Subsequent research has not been performed in vivo; therefore, an in vivo quantitative analysis is needed to determine the effective SSP stretching positions. The purpose of the present study was to quantitatively verify the effective SSP stretching positions using ultrasonic shear wave elastography in vivo. 
Materials and Methods

117 We conducted this experimental study in accordance with the Declaration of Helsinki.

\subsection{Participants}

An a priori power analysis was conducted using $G *$ Power software version 3.1 (Heinrich

Heine University, Dusseldorf, Germany). We estimated that a sample size of 14 participants was required based on a 0.25 effect size, $0.05 \alpha$ level, and 0.8 desired power level. Therefore, 15 healthy men (mean \pm standard deviation; age: $23.4 \pm 3.0$ years, height: $172.9 \pm 3.0 \mathrm{~cm}$, weight: $66.3 \pm 6.0 \mathrm{~kg}$ ) were included. Participants with a history of neuromuscular disease or musculoskeletal injury involving the upper extremities were excluded. All participants were informed of the purpose and methods of the study before providing written consent.

\subsection{Data Collection}

Shear wave speed was measured by an Aixplorer ultrasound system using an SL10-2

130 linear array transducer (Supersonic Imagine, Aix-en-Provence, France) to assess the shear elastic modulus of the SSP in the nondominant shoulder. We examined the nondominant side to determine the influence of the shoulder position on the shear elastic modulus of the SSP, because some volunteers had experience participating in overhead sports.

An ultrasound probe was placed $20 \mathrm{~mm}$ above the midpoint between the acromial angle and the root of the spine of scapula. The ultrasound images were used to align the probe parallel to the SSP muscle fiber orientation as much as possible (Figure 1). Participants were instructed to sit relaxed on a chair. To consistently position each participant, all procedures were performed by the same 3 testers. One tester measured the shear wave speed, the second fixed the participant's thorax, and the third changed the arm positions (Figure 2). To 
minimize the measurement error, the shear elastic modulus was measured twice in the same position.

As many measurement positions were selected as possible while preventing patient fatigue and confounding results from stretching. The shear elastic modulus of the SSP was measured in the 10 arm positions under the following conditions: neutral position to evaluate the effect of stretching (reference), arm positions, including horizontal adduction to compare the effect of horizontal abduction, which was recommended in a previous study, and different combinations of varying shoulder joint angles, including horizontal adduction to detect motions that emphasized SSP stretching in the 3 shoulder motions. Actual measurement positions are IR at $0^{\circ}$ elevation (Ele0), IR at $90^{\circ}$ abduction (Abd90), IR at $90^{\circ}$ flexion (Fle90), IR at maximum extension (Ext), IR at maximum horizontal adduction with $90^{\circ}$ elevation (Ele90HAd), IR at maximum horizontal adduction with $45^{\circ}$ elevation (Ele45HAd), IR at maximum horizontal abduction with $20^{\circ}$ elevation (Ele20HAb), IR at maximum horizontal abduction with $45^{\circ}$ elevation (Ele45HAb), IR at maximum horizontal abduction with $90^{\circ}$ elevation (Ele90HAb), and a neutral rotation at $0^{\circ}$ elevation (Rest).

The arm positions were defined based on the globe system ${ }^{3}$. In this study, horizontal adduction and horizontal abduction were defined as forward and backward changes of the plane of elevation. Elevation of the humerus in the $90^{\circ}, 0^{\circ}$, and $-90^{\circ}$ planes was defined as flexion, abduction, and extension, respectively. The arm positions were defined as a combination of 3 shoulder motions. The sequence in which the arm was moved into the measurement position was elevation, subsequently horizontal abduction/adduction, and lastly, rotation. For elevation, the shoulder joint was moved to $45^{\circ}$ or $90^{\circ}$ abduction, as measured by a goniometer, and this angle was fixed during the subsequent 2 motions using a mark on a vertical pole to indicate the height of the elbow. For Ele20HAb, the position was defined by moving the elbow into the horizontal abduction position (ie, toward the participant's back) 
with the elbow contacting the thorax as much as possible without necessarily maintaining the height of the elbow at $20^{\circ}$ elevation. For horizontal and rotational motion, the shoulder joint was moved to the maximum range of motion the individual could tolerate without discomfort or pain. The arm positions were performed in random order to preclude any effect of the measurement sequence

\subsection{Data Analysis}

The mean shear wave propagation speed $(\mathrm{m} / \mathrm{s})$ within the region of interest was automatically calculated. The shear elastic modulus $(G)$ can be calculated using the shear wave speed (cs) through the following equation ${ }^{29}$ :

$$
G=\rho c_{s}^{2}
$$

where $\rho$ is the muscle mass density and is assumed to be $1,000 \mathrm{~kg} / \mathrm{m}^{3}$.

Measurement reliability was assessed using the intraclass correlation coefficient (ICC 1,1$)$ with a 95\% confidence interval. Comparison of the shear elastic modulus among the measurement positions was assessed using the mean value \pm standard deviation.

A 1-way repeated measures analysis of variance was used to determine the difference in the shear elastic modulus of the SSP among the stretching positions. When a significant main effect was observed, the difference among positions was determined using the Bonferroni post hoc test. Statistical significance was defined using an $\alpha=0.05$ for all tests. Statistical analyses were performed using IBM SPSS Statistics 22.0 software (IBM, Armonk, NY, USA). 
Results

187 Reliability of the shear elastic modulus was assessed using the ICC with a 95\% confidence

188 interval (Table I). The ICC ranged from 0.81 for Ele90HAd to 0.98 for Rest. The shear elastic

189 modulus at Rest was $8.7 \pm 3.5 \mathrm{kPa}$ and moduli in other positions are provided in Table II. The

190 mean shear elastic modulus was highest at Ext, followed by Ele90HAb, Ele45HAb,

191 Ele45HAd, Abd90, Ele20HAb, Ele90HAd, Fle90, Rest, and Ele0 (Fig. 3).

192 Repeated measures analysis of variance revealed a significant effect on the shear elastic

193 modulus. Bonferroni post hoc tests indicated that the shear elastic moduli in 3 positions (Ext,

194 Ele90HAb, and Ele45HAb) were significantly greater than those in the other 7 positions (Fig.

195 3). Only these 3 positions had shear elastic moduli that were significantly greater than that at

196 Rest (Table II), and there were no significant differences in shear elastic moduli among these

1973 positions. Differences in shear elastic moduli among the other 7 positions were not 198 significant. 
199

200

201

202

203

204

205

206

207

208

209

210

211

213

\section{Discussion}

The results of this study show that the shear elastic moduli in Ext, Ele90HAb, and Ele45HAb were significantly greater than those in the other 7 positions. This suggests that these 3 positions are more effective stretching positions for the SSP than the other 7 positions. To the best of our knowledge, this is the first report to investigate the effective SSP stretching positions using quantitative analysis with ultrasonic shear wave elastography in vivo.

In this study, the ICC ranged from 0.81 to 0.98 for all positions. ICCs in this range rank as "almost perfect” reliability according to the criteria of Landis ${ }^{20}$. Therefore, we consider the data in this study reliable. The Rest position was the most reproducible position and, therefore, the shear elastic modulus in that position had the highest reliability. In contrast, the shear elastic modulus in Ele90HAd demonstrated the lowest reliability among the 10 positions.

The shear elastic moduli in Ext, Ele90HAb, and Ele45HAb were significantly greater than that in Rest, suggesting that these 3 positions are effective SSP stretching positions. In contrast, the shear elastic moduli in Ele0, Abd90, Fle90, Ele90HAd, Ele45HAd, and Ele20HAb did not differ significantly from that in Rest, suggesting that these positions are not effective SSP stretching positions. All effective stretching positions found in this study include elevation, horizontal abduction, and maximum IR.

In clinical rehabilitation and sports, the cross-body stretch and the sleeper stretch have been widely used to improve posterior shoulder tightness. These positions are similar to the Ele90HAd and Fle90 positions used in this study. However, the shear elastic moduli in Ele90HAd and Fle90 were not significantly different compared with that in Rest. Our results suggest that horizontal abduction is more important than horizontal adduction for stretching the SSP. In other words, the arm is positioned not in front but in the back of the body to stretch the SSP effectively. In previous studies, the SSP has been found to have an IR 
moment arm at $90^{\circ}$ of humeral elevation in the sagittal plane ${ }^{17}$. In the position in which SSP has an IR moment arm, contraction of SSP leads to IR. In other words, to stretch the SSP at $90^{\circ}$ humeral elevation in the sagittal plane, the humerus must be externally rotated, not internally rotated.

Because differences in the shear elastic moduli among Ext, Ele90HAb, and Ele45HAb were not significant, we could not identify the most effective stretching position. The shear elastic moduli in Ext, Ele90HAb, and Ele45HAb were significantly greater than that in Ele20HAb. It is likely that higher elevation of the humerus behind the body is important to stretch the SSP.

Some of the following SSP stretching positions have been recommended: fully adducting the arm behind the back $^{6}$, positioning the arm behind the back while maintaining medial rotation $^{10}$, and placing the hand behind the back and reaching up between the shoulder blades $^{31}$. In terms of the distance between the elbow and the back, these 3 recommended positions are similar to the Ele20HAb position used in this study. Judging from the results of this study, these 3 positions need to emphasize elevation to effectively stretch the SSP.

239 Previous studies evaluated other factors, such as muscle contraction, pressure to the muscle, traction on the bone, and posture of the whole body, in addition to shoulder position. These factors may influence the effect of stretching. In contrast, Ylinen ${ }^{36}$ recommended extension, adduction, and IR. When compared with elevation, this position is an effective SSP stretch. In terms of the importance of elevation and horizontal abduction, our results are similar to those of the quantitative analysis using cadavers reported by Muraki et al. ${ }^{24}$ All of our test positions, except for Rest, included maximum IR. Whether maximum IR is necessary or not will require further study. young men. Sex, age, and differences in sport and disease experience may affect the shear 
249 elastic modulus of the shoulder muscles; therefore, we need to be careful when using these 250 results for therapy and training in clinical or athletic settings.

251 Second, we allowed the free movement of the scapula when positioning the humerus.

252 Measuring the movement of the scapula may produce more accurate results. In addition to the 253 shoulder position, most of the previous authors examined muscle contraction, pressure to the 254 muscle, traction on the bone, and posture of the whole body. However, the focus of the 255 current study was to evaluate the influence of the shoulder position only. Examining the 256 influence of these other factors will be necessary in future studies.

257 We only evaluated the SSP in this study. Further measurements targeting the infraspinatus 258 and other muscles should be conducted. By clarifying effective positions in multiple muscles, 259 we may be able to determine the position needed to stretch multiple muscles simultaneously 260 or to selectively stretch 1 muscle. 


\section{Conclusions}

263 This study used quantitative analysis to determine the effective stretching positions for the 264 SSP muscle, using ultrasonic shear wave elastography in vivo. Our results suggest that 265 maximum internal rotation at maximum extension, maximum internal rotation at maximum 266 horizontal abduction with $90^{\circ}$ elevation, and maximum internal rotation at maximum 267 horizontal abduction with $45^{\circ}$ elevation are effective stretching positions for the SSP muscle. 


\section{References}

269 1. Bercoff J, Tanter M, Fink M. Supersonic shear imaging: a new technique for soft tissue elasticity mapping. IEEE Trans Ultrason Ferroelectr Freq Control 2004;51:396-409.

2. Buneo CA, Soechting JF, Flanders M. Postural dependence of muscle actions: implications for neural control. J Neurosci 1997;17:2128-2142.

5. Eismann EA, Little KJ, Laor T, Cornwall R. Glenohumeral abduction contracture in

3. Doorenbosch CA, Harlaar J, Veeger DH. The globe system: an unambiguous description of shoulder positions in daily life movements. J Rehabil Res Dev 2003;40:147-155.

6. Evjenth O, Hamberg J. Muscle Stretching in Manual Therapy: A Clinical Manual: The

7. Freitas SR, Andrade RJ, Larcoupaille L, Mil-homens P, Nordez A. Muscle and joint responses during and after static stretching performed at different intensities. Eur J Appl Physiol 2015;115:1263-1272. doi: 10.1007/s00421-015-3104-1

8. Hatta T, Giambini H, Uehara K, Okamoto S, Chen S, Sperling JW, et al. Quantitative assessment of rotator cuff muscle elasticity: Reliability and feasibility of shear wave elastography. J Biomech 2015;48:3853-3858. doi: 10.1016/j.jbiomech.2015.09.038

10. Houglum PA. Therapeutic exercise for musculoskeletal injuries. Champaign, IL: Human Kinetics; 2010. (ISBN No. 9780736075954)

11. Ichihashi N, Umegaki H, Ikezoe T, Nakamura M, Nishishita S, Fujita K, et al. The effects of a 4 -week static stretching programme on the individual muscles comprising the hamstrings. J Sports Sci 2016;34:2155-2159. doi: 10.1080/02640414.2016.1172725

12. Itoigawa Y, Sperling JW, Steinmann SP, Chen Q, Song P, Chen S, et al. Feasibility assessment of shear wave elastography to rotator cuff muscle. Clin Anat 2015;28:213-218. doi: $10.1002 /$ ca.22498 passive muscle force: an ex-vivo study. J Biomech 2013;46:2053-2059. doi: 
306

307

308

309

310

311

312

313

314

315

316

317

318

319

320

321

322

323

324

325

326

327

328

329

330

331

332

333

334

335

336

337

338

339

340

341

342

343

15. Koo TK, Guo JY, Cohen JH, Parker KJ. Quantifying the passive stretching response of human tibialis anterior muscle using shear wave elastography. Clin Biomech (Bristol, Avon) 2014;29:33-39. doi: 10.1016/j.clinbiomech.2013.11.009

16. Kuechle DK, Newman SR, Itoi E, Morrey BF, An KN. Shoulder muscle moment arms during horizontal flexion and elevation. J Shoulder Elbow Surg 1997;6:429-439.

17. Kuechle DK, Newman SR, Itoi E, Niebur GL, Morrey BF, An KN. The relevance of the moment arm of shoulder muscles with respect to axial rotation of the glenohumeral joint in four positions. Clin Biomech (Bristol, Avon) 2000;15:322-329.

18. Kusano K, Nishishita S, Nakamura M, Tanaka H, Umehara J, Ichihashi N. Acute effect and time course of extension and internal rotation stretching of the shoulder on infraspinatus muscle hardness. J Shoulder Elbow Surg 2017;26:1782-1788. doi: 10.1016/j.jse.2017.04.018

19. Lacourpaille L, Hug F, Bouillard K, Hogrel JY, Nordez A. Supersonic shear imaging provides a reliable measurement of resting muscle shear elastic modulus. Physiol Meas 2012;33:N19-N28. doi: 10.1088/0967-3334/33/3/N19

20. Landis JR, Koch GG. The measurement of observer agreement for categorical data. Biometrics 1977;33:159-174.

21. Leong HT, Ng GY, Leung VY, Fu SN. Quantitative estimation of muscle shear elastic modulus of the upper trapezius with supersonic shear imaging during arm positioning. PLoS One 2013;8:e67199. doi: 10.1371/journal.pone.0067199

22. McClure P, Balaicuis J, Heiland D, Broersma ME, Thorndike CK, Wood A. A randomized controlled comparison of stretching procedures for posterior shoulder tightness. J Orthop Sports Phys Ther 2007;37:108-114. doi: 10.2519/jospt.2007.2337

23. Muraki T, Aoki M, Izumi T, Fujii M, Hidaka E, Miyamoto S. Lengthening of the pectoralis minor muscle during passive shoulder motions and stretching techniques: a cadaveric biomechanical study. Phys Ther 2009;89:333-341. doi: 10.2522/ptj.20080248

24. Muraki T, Aoki M, Uchiyama E, Murakami G, Miyamoto S. The effect of arm position on stretching of the supraspinatus, infraspinatus, and posterior portion of deltoid muscles: a cadaveric study. Clin Biomech (Bristol, Avon) 2006;21:474-480. doi: 10.1016/j.clinbiomech.2005.12.014

25. Muraki T, Aoki M, Uchiyama E, Takasaki H, Murakami G, Miyamoto S. A cadaveric study of strain on the subscapularis muscle. Arch Phys Med Rehabil 2007;88:941-946. doi: 10.1016/j.apmr.2007.04.003

26. Nakamura M, Ikezoe T, Umegaki H, Kobayashi T, Nishishita S, Ichihashi N. Changes in Passive Properties of the Gastrocnemius Muscle-Tendon Unit During a 4-Week Routine Static Stretching Program. J Sport Rehabil 2017;26:263-268. doi: 10.1123/jsr.2015-0198

27. Nordez A, McNair PJ, Casari P, Cornu C. Static and cyclic stretching: their different effects on the passive torque-angle curve. J Sci Med Sport 2010;13:156-160. doi: 
10.1016/j.jsams.2009.02.003

28. Rosskopf AB, Ehrmann C, Buck FM, Gerber C, Fluck M, Pfirrmann CW. Quantitative Shear-Wave US Elastography of the Supraspinatus Muscle: Reliability of the Method and Relation to Tendon Integrity and Muscle Quality. Radiology 2016;278:465-474. doi: 10.1148/radiol.2015150908

29. Shiina T, Nightingale KR, Palmeri ML, Hall TJ, Bamber JC, Barr RG, et al. WFUMB guidelines and recommendations for clinical use of ultrasound elastography: Part 1: basic principles and terminology. Ultrasound Med Biol 2015;41:1126-1147. doi: 10.1016/j.ultrasmedbio.2015.03.009

30. Umehara J, Nakamura M, Fujita K, Kusano K, Nishishita S, Araki K, et al. Shoulder horizontal abduction stretching effectively increases shear elastic modulus of pectoralis minor muscle. J Shoulder Elbow Surg 2017;26:1159-1165. doi: 10.1016/j.jse.2016.12.074

31. Walker B. The anatomy of stretching : your illustrated guide to flexibility and injury rehabilitation. Berkeley, Calif.: North Atlantic Books; 2011. (ISBN No. 9781583943717)

32. Webb JD, Blemker SS, Delp SL. 3D finite element models of shoulder muscles for computing lines of actions and moment arms. Comput Methods Biomech Biomed Engin 2014;17:829-837. doi: 10.1080/10255842.2012.719605

33. Weiss PL, Kearney RE, Hunter IW. Position dependence of ankle joint dynamics--I. Passive mechanics. J Biomech 1986;19:727-735.

34. Weppler CH, Magnusson SP. Increasing muscle extensibility: a matter of increasing length or modifying sensation? Phys Ther 2010;90:438-449. doi: 10.2522/ptj.20090012

35. Wilk KE, Meister K, Andrews JR. Current concepts in the rehabilitation of the overhead throwing athlete. Am J Sports Med 2002;30:136-151. doi: 10.1177/03635465020300011201

36. Ylinen J. Stretching Therapy: For Sport and Manual Therapies. London, United Kingdom: Churchill Livingstone; 2008. (ISBN No. 9780443101274)

37. Yoshida N, Domen K, Koike Y, Kawato M. A method for estimating torque-vector directions of shoulder muscles using surface EMGs. Biol Cybern 2002;86:167-177. doi: $10.1007 / \mathrm{s} 00422-001-0286-\mathrm{x}$ 
$373 \quad$ Figure and Table Legends.

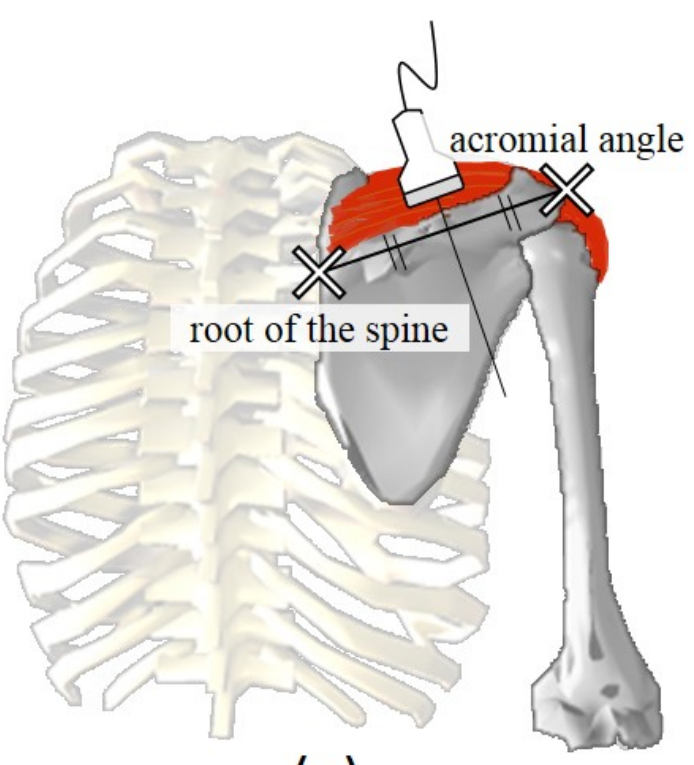

(a)

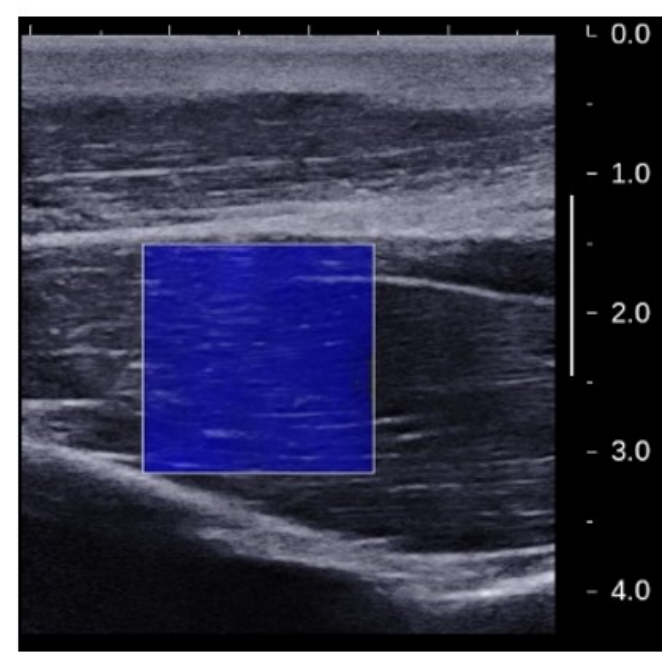

(b)

374

375 Figure 1 Position and angle of the probe during measurement. (a) An ultrasound probe was

376 placed $20 \mathrm{~mm}$ above the midpoint between the acromial angle and the root of the spine of

377 scapula. (b) The ultrasound images were used to align the probe parallel to the supraspinatus

378 muscle fiber orientation as much as possible. 


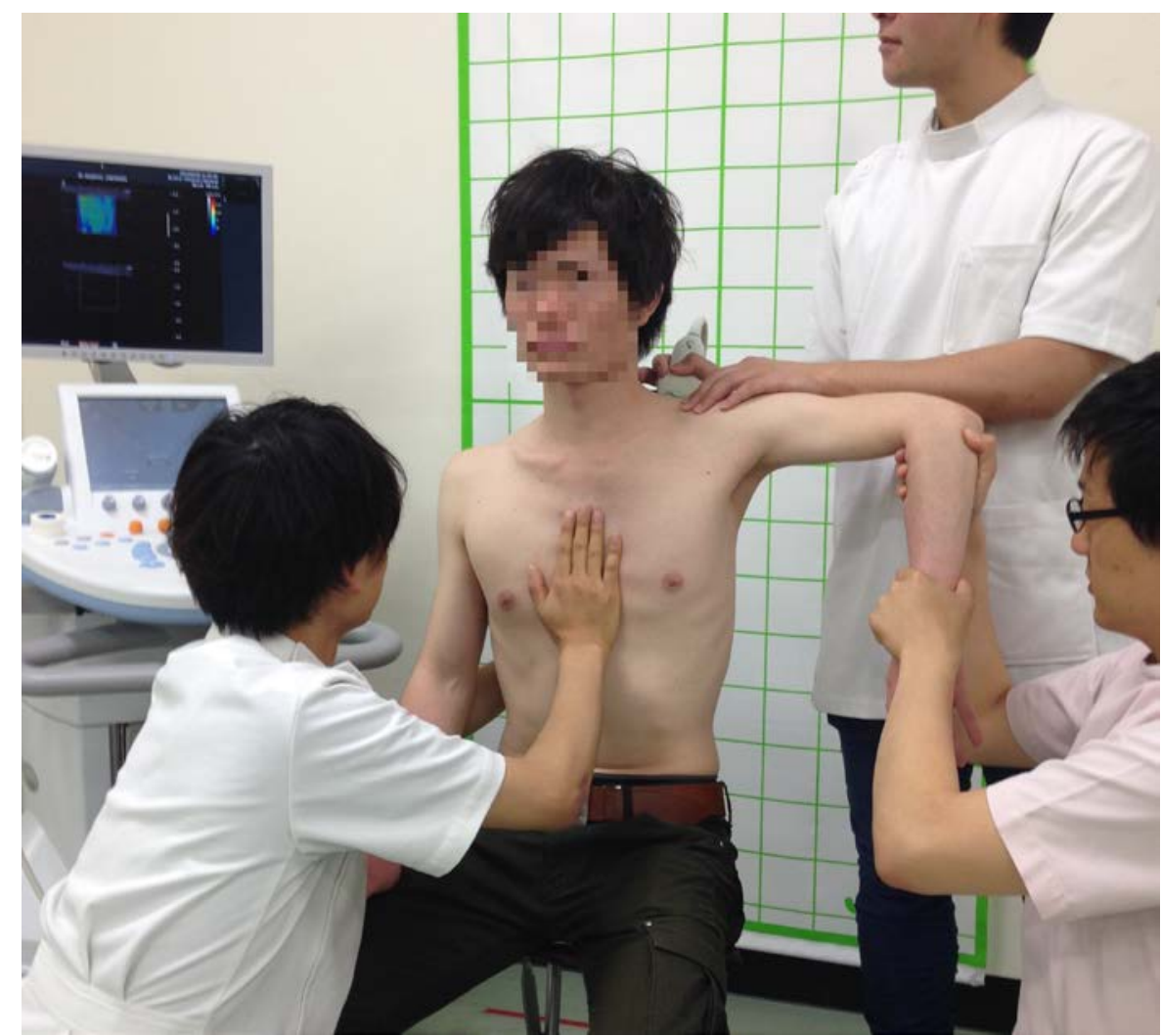

381 Figure 2 Experimental setup. Participants were instructed to sit relaxed on a chair. To

382 consistently position each participant, all procedures were performed by the same 3 testers:

383 the first tester measured the shear wave speed, the second fixed the participant's thorax, and 384 the third changed the arm positions. 


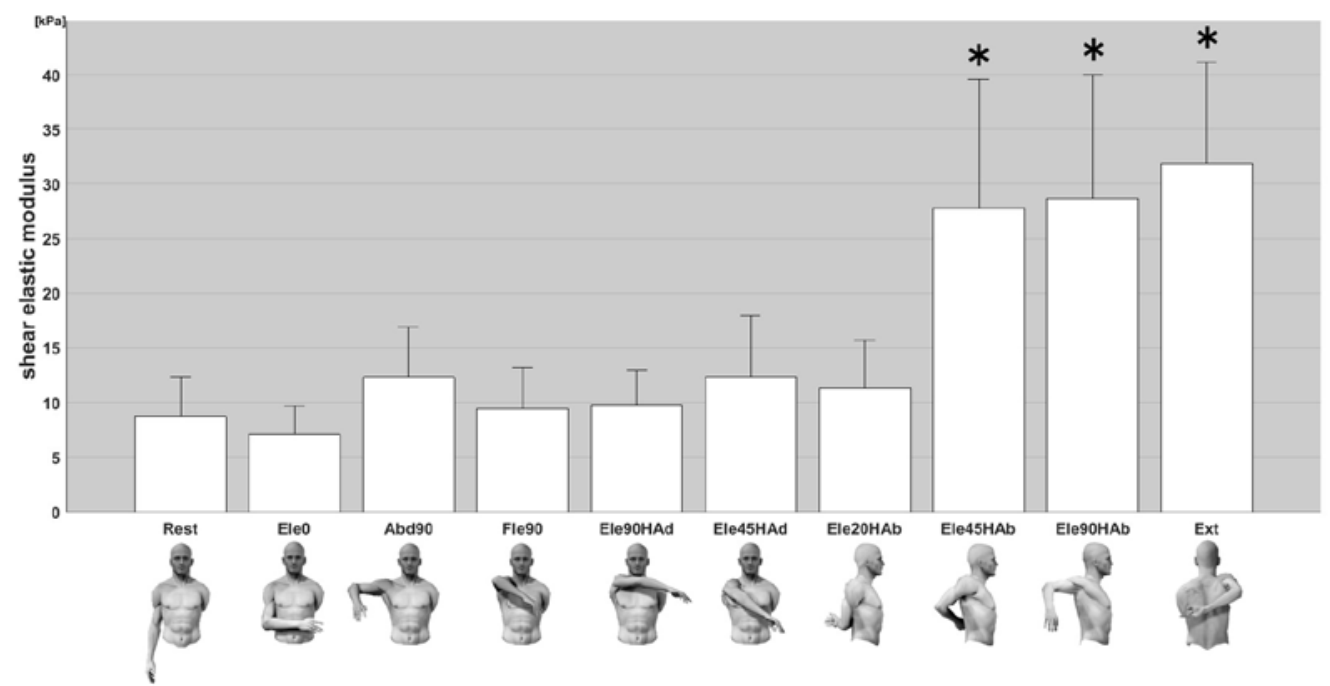

386

387 Figure 3 Shear elastic moduli of the supraspinatus muscle in each measurement position. The error bar shows the standard deviation. *Indicates that the shear elastic moduli in Ext, Ele90HAb, and Ele45HAb were significantly greater than those in the other 7 positions: Ele45HAb-Rest $\quad(\mathrm{P}=.002), \quad$ Ele45HAb-Abd90 $\quad(\mathrm{P}=.011), \quad$ Ele45HAb-Fle90 $\quad(\mathrm{P}=.005)$, Ele45HAb-Ele90HAd $(\mathrm{P}=.002)$, Ele45HAb-Ele45HAd $(\mathrm{P}=.047)$, Ele45HAb-Ele20HAb $(\mathrm{P}=.007), \quad$ Ele90HAb-Rest $\quad(\mathrm{P}=.001), \quad$ Ele90HAb-Abd90 $\quad(\mathrm{P}=.013), \quad$ Ele90HAb-Fle90 $(\mathrm{P}=.001)$, Ele90HAb-Ele90HAd $(\mathrm{P}=.001)$, Ele90HAb-Ele20HAd $(\mathrm{P}=.002)$, and the other positions $(\mathrm{P}<.001)$. Rest, neutral rotation at $0^{\circ}$ elevation; Ele0, maximum internal rotation at $0^{\circ}$ elevation; Abd90, maximum internal rotation at $90^{\circ}$ abduction; Fle90, maximum internal rotation at $90^{\circ}$ flexion; Ele90Had, maximum internal rotation at maximum horizontal adduction with $90^{\circ}$ elevation; Ele45Had, maximum internal rotation at maximum horizontal adduction with $45^{\circ}$ elevation; Ele20HAb, maximum internal rotation at maximum horizontal abduction with $20^{\circ}$ elevation; Ele45HAb, maximum internal rotation at maximum horizontal abduction with $45^{\circ}$ elevation, Ele90HAb, maximum internal rotation at maximum horizontal abduction with $90^{\circ}$ elevation; Ext, maximum internal rotation at maximum extension. 
403 Table I Intraclass correlation coefficient in each measurement position.

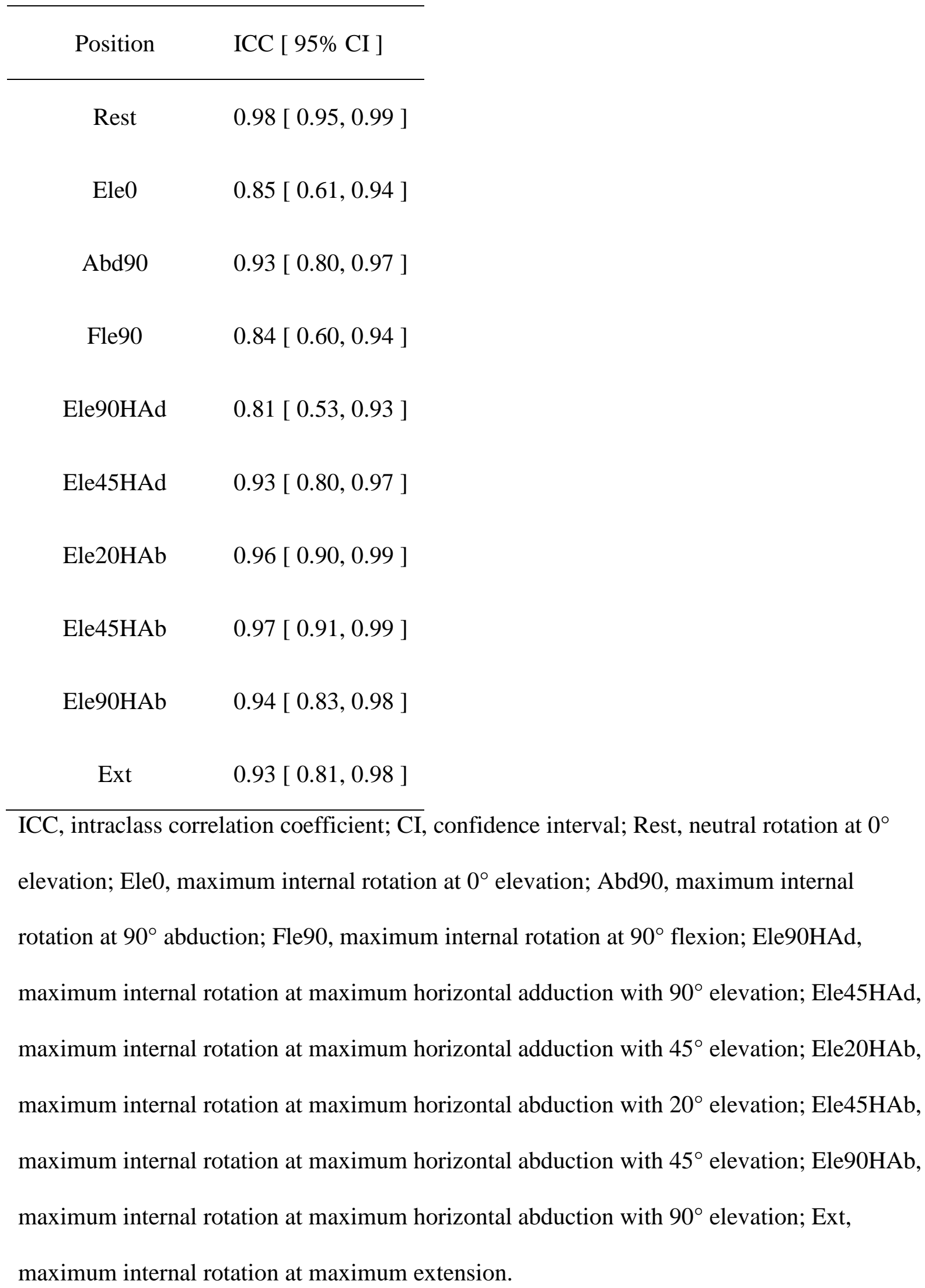


414 Table II Shear elastic modulus of the supraspinatus muscle in each measurement position.

\begin{tabular}{lcc}
\hline \multicolumn{1}{l}{ Position } & Mean \pm S.D. [kPa] & p value (Comparison with Rest) \\
\hline Rest & $8.7 \pm 3.5$ & - \\
Ele0 & $7.1 \pm 2.6$ & $>0.999$ \\
Abd90 & $12.3 \pm 4.4$ & 0.725 \\
Fle90 & $9.4 \pm 3.6$ & $>0.999$ \\
Ele90HAd & $9.8 \pm 3.0$ & $>0.999$ \\
Ele45HAd & $12.4 \pm 5.4$ & $>0.999$ \\
Ele20HAb & $11.3 \pm 4.2$ & $>0.999$ \\
Ele45HAb & $31.9 \pm 8.9$ & 0.002 \\
Ele90HAb & $27.8 \pm 11.4$ & 0.001 \\
Ext & $28.7 \pm 11.0$ & $<0.001$ \\
\hline
\end{tabular}

SD, standard deviation; Rest, neutral rotation at $0^{\circ}$ elevation; Ele0, maximum internal

416 rotation at $0^{\circ}$ elevation; Abd90, maximum internal rotation at $90^{\circ}$ abduction; Fle90, 417 maximum internal rotation at $90^{\circ}$ flexion; Ele90HAd, maximum internal rotation at 418 maximum horizontal adduction with $90^{\circ}$ elevation; Ele45HAd, maximum internal rotation at 419 maximum horizontal adduction with $45^{\circ}$ elevation; Ele20HAb, maximum internal rotation at 420 maximum horizontal abduction with $20^{\circ}$ elevation; Ele45HAb, maximum internal rotation at 421 maximum horizontal abduction with $45^{\circ}$ elevation; Ele90HAb, maximum internal rotation at 422 maximum horizontal abduction with $90^{\circ}$ elevation; Ext, maximum internal rotation at 423 maximum extension. 